\title{
Dynamical Model of an Electromagnet using Cauer Ladder Network Representation of Eddy-current Fields
}

\author{
Yuji Shindo $^{* a)} \quad$ Member, Akihisa Kameari* ${ }^{* *}$ Member \\ Kengo Sugahara $^{* * *}$ Member, Tetsuji Matsuo ${ }^{* * * *}$ Member
}

(Manuscript received Oct. 12, 2017, revised Jan. 15, 2018)

\begin{abstract}
This paper presents a novel dynamical modeling method for magnetic actuators. The magnetic field is decomposed into orthogonal distribution functions by using the Cauer ladder network representation of eddy-current fields. The linearized transfer function of the magnetic force is derived by using the Maxwell's stress tensor and distribution functions. The numerical example of an electromagnet is presented to show the effectiveness of this method. It is revealed that the eddy current not only reduces the main flux but also induces a repulsive force at the air gap. The details of the Cauer ladder network representations are also clarified.
\end{abstract}

Keywords: current stiffness, equivalent circuit, Neumann series, Krylov subspace, Parseval's equality, Gram-Schmidt orthogonalization

\section{Introduction}

The eddy current in the core of electromagnet yields the response lag of the actuators. It is remarkable if the magnet employs solid materials for magnetic circuits. If one attempts to estimate the entire system as Fig. 1, finite element (FE) analyses become the bottleneck for calculation. Thus the dynamical modeling is important to estimate the response of the actuators, especially in the area of magnetic levitations ${ }^{(1)}$, where the quick response of the actuator is required.

Two approaches are popular to derive the dynamics of the electromagnet. One is analytical modeling by using the equivalent magnetic circuit, which is preferred frequently ${ }^{(2)(3)}$ because of its ease. The other is the identification of the transfer function of the electromagnet by using the FE analyses ${ }^{(4)}$. However, the identifications over the entire frequency range may require much time and effort.

Recently, an effective modeling method for eddy-current field is proposed. This method adopts the Cauer ladder network (CLN) as an equivalent circuit of the eddy-current field ${ }^{(5)}$. There, the magnetic field can be represented by linear combinations of distribution functions, obtained by FE static analyses performed beforehand. The coefficients of the linear combinations are voltage or current variables of the equivalent circuit. Although this method can be regarded as an enhancement of one-dimensional modeling method using orthogonal polynomials ${ }^{(6)(7)}$, the usability is much extended

a) Correspondence to: Yuji Shindo. E-mail: shindo_yuji@khi. co.jp

* Kawasaki Heavy Industries, Ltd.

1-1, Kawasaki-cho, Akashi 673-8666, Japan

** Science Solutions International Laboratory, Inc.

2-21-7, Nakamachi, Meguro-ku, Tokyo 153-0065, Japan

*** Faculty of Science and Engineering, Kindai University 3-4-1, Kowakae, Higashiosaka, Osaka 577-8502, Japan

**** Graduate School of Engineering, Kyoto University

Nishikyo-ku, Kyoto 615-8530, Japan

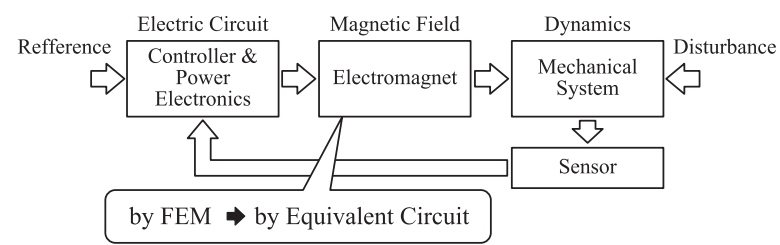

Fig. 1. Typical control system

because it can be applied to arbitrary eddy-current field.

The benefits of this method are summarized as the followings. First, the transient FE analysis is transformed to an equivalent circuit analysis. This provides remarkable reduction of computations. Second, the magnetic field at any point of time can be reconstructed by using the distribution functions and circuit variables. Third, although this method is basically derived by the FE linear analyses, it can deal with the nonlinear hysteresis materials by adopting the nonlinear hysteresis inductors in the equivalent circuit $^{(8)(9)}$.

This paper shows that a dynamic model of the magnetic force of an electromagnet can be derived by using the CLN representation, when the materials are assumed to be linear. The magnetic force when the air gap kept constant and the exciting current fluctuates is considered here. It is sometimes called current stiffness. It is shown that the generic dynamical magnetic force is represented by the quadratic form of the currents in the CLN. The linearized transfer function under a bias current is derived. The frequency responses of an electromagnet are obtained and compared with the existent FE analysis. It is also revealed that the eddy-current not only reduce the main flux, but also induce the repulsive force at the air gap. This insight is explicitly recognized by the distribution functions of the CLN.

The minimum but sufficient proof for the CLN representation is already given in Ref. (5), where it is assumed implicitly that any eddy-current field should be transformed to 


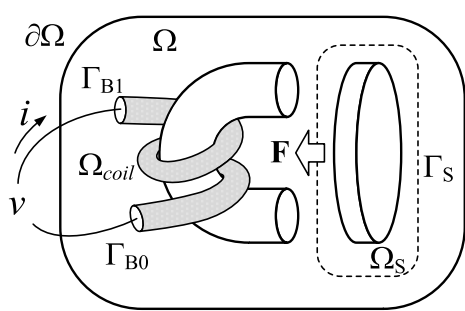

Fig. 2. Eddy-current field with an electromagnet

a ladder network. However, it is worth to reveal the details and backgrounds of this method. It is shown that this method can be derived without such assumption. The Neumann series of the differential operator ${ }^{(10)(11)}$ for the eddy-current field, the separation of variables and Parseval's equality are used there. Furthermore, it is shown that this method is related to the Krylov subspace methods ${ }^{(12)}$, often used for solving large scale linear systems and optimization problems.

\section{Cauer Ladder Network Representation}

2.1 Neumann Series for Eddy-current Field A typical eddy-current field, sometimes called magneto-dynamic field $^{(13)}$, is illustrated in Fig. 2. The electromagnet with excitation winding is contained in the entire domain. $\Omega$, surrounded by the boundary $\partial \Omega$. Equations for eddy-current field, are given by the followings, where the magnetic permeability $\mu$ and the electric conductivity $\sigma$ are assumed to be linear.

$$
\begin{aligned}
& \nabla \times \boldsymbol{H}-\sigma \boldsymbol{E}=0 \ldots \ldots \\
& \nabla \times \boldsymbol{E}+\mu \partial \boldsymbol{H} / \partial t=0 \\
& \nabla \cdot \boldsymbol{B}=0 \ldots \ldots \ldots \ldots \\
& \nabla \cdot \boldsymbol{J}=0 \cdots \cdots \cdots \\
& \boldsymbol{B}=\mu \boldsymbol{H}, \quad \boldsymbol{J}=\sigma \boldsymbol{E}, \cdots
\end{aligned}
$$

The area for conducting wire is conveniently defined as $\Omega_{\text {coil }}$, which has the surfaces of electrodes $\Gamma_{\mathrm{B} 1}$ and $\Gamma_{\mathrm{B} 2}$. The applied winding voltage $v$ is the potential difference between $\Gamma_{\mathrm{B} 0}$ and $\Gamma_{\mathrm{B} 1}$. Thus the following equations for electric potential $\phi$ are assumed.

$$
\left.\phi\right|_{\Gamma_{\mathrm{B} 1}}-\left.\phi\right|_{\Gamma_{\mathrm{B} 0}}=
$$

By putting $\partial \boldsymbol{H} / \partial t=0$ in (2) and using (4), Laplace type static electric equation $\nabla \cdot(\sigma \nabla \phi)=0$ and its solution $\boldsymbol{E}_{0}=-\nabla \phi$ are derived. Then the Joule loss $P_{0}$, resistance $R_{0}$ and the current $i_{0}$ on the electrodes $\Gamma_{\mathrm{B} 0}$ or $\Gamma_{\mathrm{B} 1}$ are given by the followings, where $\overline{\boldsymbol{E}}_{0}=\boldsymbol{E}_{0} / v$ is a vector valued distribution function having the unit $1 / \mathrm{m}$ and $\boldsymbol{n}$ is the outward normal vector on $\Gamma_{\mathrm{B} 0}$ or $\Gamma_{\mathrm{B} 1}$. From these relations, the equivalent circuit for the static electric field can be represented by Fig. 3(a).

$$
\begin{aligned}
& W_{0}=\left\langle\sigma \boldsymbol{E}_{0}, \boldsymbol{E}_{0}\right\rangle=v^{2} / R_{0} \ldots \ldots \ldots \ldots \ldots \ldots \ldots \\
& 1 / R_{0}=\left\langle\sigma \overline{\boldsymbol{E}}_{0}, \overline{\boldsymbol{E}}_{0}\right\rangle \\
& i=v / R_{0}=-\int_{\Gamma_{\mathrm{B} 0}} \boldsymbol{E}_{0} \cdot \boldsymbol{n} \mathrm{d} S=\int_{\Gamma_{\mathrm{B} 1}} \boldsymbol{E}_{0} \cdot \boldsymbol{n} \mathrm{d} S \cdots \cdots
\end{aligned}
$$

Here, the inner product in domain $\Omega$ is defined as the following.
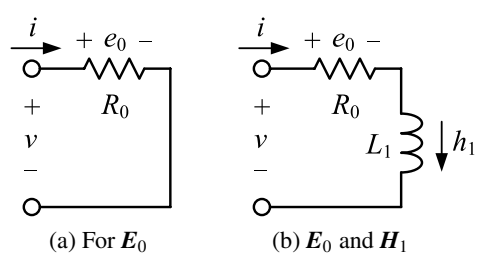

Fig. 3. Generation of static equivalent circuit

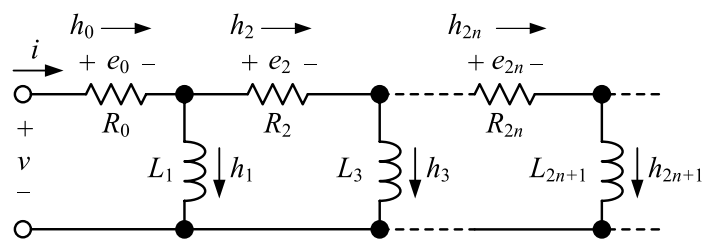

Fig. 4. Infinite Cauer ladder network

$$
\langle\boldsymbol{X}, \boldsymbol{Y}\rangle=\int_{\Omega} \boldsymbol{X} \cdot \boldsymbol{Y} \mathrm{d} V
$$

The electric field $\boldsymbol{E}_{0}$ generates the current $\boldsymbol{J}_{0}=\sigma \boldsymbol{E}_{0}$ and magnetic field $\boldsymbol{H}_{1}$ by solving (1). By setting $\overline{\boldsymbol{H}}_{1}=\boldsymbol{H}_{1} / i$, the magnetic energy and the DC inductance is given by the followings. Then the DC equivalent circuit Fig. 3(b) is obtained.

$$
\begin{aligned}
& W_{1}=\left\langle\mu \boldsymbol{H}_{1}, \boldsymbol{H}_{1}\right\rangle / 2=L_{1} i^{2} / 2 \\
& L_{1}=\left\langle\mu \overline{\boldsymbol{H}}_{1}, \overline{\boldsymbol{H}}_{1}\right\rangle \ldots \ldots \ldots \ldots
\end{aligned}
$$

The Cauer ladder network representation, shown in Fig. 4, is a natural and complete expansion of this discussion to the eddy-current field. For the sake of argument, following vector spaces are defined. Hereafter, the electric field $\boldsymbol{E}$ and magnetic field $\boldsymbol{H}$ are assumed to belong $\mathcal{E}$ and $\mathcal{H}$ respectively.

$$
\begin{aligned}
& \mathcal{E}=\left\{\boldsymbol{E} \in \mathrm{C}^{3} \mid \nabla \cdot(\sigma \boldsymbol{E})=0\right\} \\
& \mathcal{H}=\left\{\boldsymbol{H} \in \mathrm{C}^{3} \mid \nabla \cdot(\mu \boldsymbol{H})=0\right\},
\end{aligned}
$$

The alternative form of (1) and (2) is expressed by the matrix form as (14), which is the mapping from the product space $\mathcal{E} \times \mathcal{H}$ to itself. Here the time differential operator is substituted by $\mathrm{j} \omega$, and $\rho=1 / \sigma, v=1 / \mu$ are the electric and magnetic resistivity respectively.

$$
\left[\begin{array}{cc}
-1 & \rho \nabla \times \\
v \nabla \times & \mathrm{j} \omega
\end{array}\right]\left[\begin{array}{l}
\boldsymbol{E} \\
\boldsymbol{H}
\end{array}\right]=\left[\begin{array}{l}
0 \\
0
\end{array}\right]
$$

By setting $\omega=0$ in (14), the following equation for the static field is defined. Here, the electric field $\boldsymbol{E}_{0}$ and magnetic field $\boldsymbol{H}_{1}$ coincide to those discussed above.

$$
\left[\begin{array}{cc}
-1 & \rho \nabla \times \\
v \nabla \times & 0
\end{array}\right]\left[\begin{array}{l}
\boldsymbol{E}_{0} \\
\boldsymbol{H}_{1}
\end{array}\right]=\left[\begin{array}{l}
0 \\
0
\end{array}\right]
$$

From (14) and (15), the following remainder, the right side of (16), is derived.

$$
\left[\begin{array}{cc}
-1 & \rho \nabla \times \\
v \nabla \times & \mathrm{j} \omega
\end{array}\right]\left[\begin{array}{c}
\boldsymbol{E}-\boldsymbol{E}_{0} \\
\boldsymbol{H}-\boldsymbol{H}_{1}
\end{array}\right]=-\left[\begin{array}{c}
0 \\
\mathrm{j} \omega \boldsymbol{H}_{1}
\end{array}\right]
$$

Next, solve following equation.

$$
\left[\begin{array}{cc}
-1 & \rho \nabla \times \\
v \nabla \times & 0
\end{array}\right]\left[\begin{array}{l}
\boldsymbol{E}_{2} \\
\boldsymbol{H}_{3}
\end{array}\right]=-\left[\begin{array}{c}
0 \\
\mathrm{j} \omega \boldsymbol{H}_{1}
\end{array}\right] \cdots \ldots \ldots \ldots \ldots \ldots
$$


Again, we obtain the following remainder.

$$
\left[\begin{array}{cc}
-1 & \rho \nabla \times \\
v \nabla \times & \mathrm{j} \omega
\end{array}\right]\left[\begin{array}{c}
\boldsymbol{E}-\boldsymbol{E}_{0}-\boldsymbol{E}_{2} \\
\boldsymbol{H}-\boldsymbol{H}_{1}-\boldsymbol{H}_{3}
\end{array}\right]=-\left[\begin{array}{c}
0 \\
\mathrm{j} \omega \boldsymbol{H}_{3}
\end{array}\right] \ldots \ldots
$$

Repeating the above procedure, we obtain the followings if the remainder $\mathrm{j} \omega \mu \boldsymbol{H}_{2 n+1}$ converges to zero.

$$
\begin{aligned}
& \boldsymbol{E}=\boldsymbol{E}_{0}+\boldsymbol{E}_{2}+\boldsymbol{E}_{4}+\ldots \\
& \boldsymbol{H}=\boldsymbol{H}_{1}+\boldsymbol{H}_{3}+\boldsymbol{H}_{5}+\ldots
\end{aligned}
$$

The equation for the remainder (17) can be generalized by the following.

$$
\begin{aligned}
& {\left[\begin{array}{cc}
-1 & \rho \nabla \times \\
v \nabla \times & 0
\end{array}\right]\left[\begin{array}{c}
\boldsymbol{E}_{2 n} \\
\boldsymbol{H}_{2 n+1}
\end{array}\right]=\mathrm{j} \omega\left[\begin{array}{cc}
0 & 0 \\
0 & -1
\end{array}\right]\left[\begin{array}{l}
\boldsymbol{E}_{2 n-2} \\
\boldsymbol{H}_{2 n-1}
\end{array}\right] \ldots \ldots} \\
& {\left[\begin{array}{c}
\boldsymbol{E}_{2 n} \\
\boldsymbol{H}_{2 n+1}
\end{array}\right]=\mathrm{j} \omega \mathcal{A}\left[\begin{array}{l}
\boldsymbol{E}_{2 n-2} \\
\boldsymbol{H}_{2 n-1}
\end{array}\right] \ldots \ldots \ldots \ldots \ldots \ldots \ldots \ldots}
\end{aligned}
$$

Here, $\mathcal{A}$ is a differential operator defined by (22), where the last side of (22) is derived by using the formula for the inverse matrix conveniently.

$$
\mathcal{A}=\left[\begin{array}{cc}
-1 & \rho \nabla \times \\
v \nabla \times & 0
\end{array}\right]^{-1}\left[\begin{array}{cc}
0 & 0 \\
0 & -1
\end{array}\right]=\left[\begin{array}{cc}
0 & -(\nu \nabla \times)^{-1} \\
0 & -\sigma(\nabla \times)^{-1}(\nu \nabla \times)^{-1}
\end{array}\right]
$$

Note that from (22), the functions $\boldsymbol{E}_{2 n}$ and $\boldsymbol{H}_{2 n+1}$ can be solved alternately as the followings.

$$
\begin{aligned}
\boldsymbol{E}_{2 n} & =-(v \nabla \times)^{-1} \boldsymbol{H}_{2 n-1}=-(\nabla \times)^{-1} \mu \boldsymbol{H}_{2 n-1} \\
& \Leftrightarrow \nabla \times \boldsymbol{E}_{2 n}=-\mu \boldsymbol{H}_{2 n-1} \ldots \ldots \ldots \ldots \ldots \\
\boldsymbol{H}_{2 n+1} & =(\rho \nabla \times)^{-1} \boldsymbol{E}_{2 n}=(\nabla \times)^{-1} \sigma \boldsymbol{E}_{2 n} \\
& \Leftrightarrow \nabla \times \boldsymbol{H}_{2 n+1}=\sigma \boldsymbol{E}_{2 n} \ldots \ldots \ldots \ldots \ldots
\end{aligned}
$$

From (22), the Neumann series expansion of the eddycurrent field is derived as the following. The derivation carried out here gives important insight for the configuration of equivalent circuit of eddy-current field, which is discussed later.

$$
\left[\begin{array}{c}
\boldsymbol{E} \\
\boldsymbol{H}
\end{array}\right]=\left[\begin{array}{c}
\boldsymbol{E}_{0}+\boldsymbol{E}_{2}+\boldsymbol{E}_{4}+\ldots \\
\boldsymbol{H}_{1}+\boldsymbol{H}_{3}+\boldsymbol{H}_{5}+\ldots
\end{array}\right]=\sum_{n=0}^{\infty}(\mathrm{j} \omega)^{n} \mathcal{A}^{n}\left[\begin{array}{l}
\boldsymbol{E}_{0} \\
\boldsymbol{H}_{1}
\end{array}\right]
$$

It is worth to note that all the functions $\left[\boldsymbol{E}_{2 n} \boldsymbol{H}_{2 n+1}\right]^{\mathrm{T}}(n=$ $0,1, \ldots)$ and the solution $\left[\begin{array}{ll}\boldsymbol{E} & \boldsymbol{H}\end{array}\right]^{\mathrm{T}}$ of (14) belong to Krylov subspace $\mathcal{K}$ in $\mathcal{E} \times \mathcal{H}$, which is defined by the following.

$$
\mathcal{K}\left\{\mathcal{A},\left[\begin{array}{l}
\boldsymbol{E}_{0} \\
\boldsymbol{H}_{1}
\end{array}\right]\right\}=\operatorname{span}\left\{\left[\begin{array}{l}
\boldsymbol{E}_{0} \\
\boldsymbol{H}_{1}
\end{array}\right], \mathcal{A}\left[\begin{array}{l}
\boldsymbol{E}_{0} \\
\boldsymbol{H}_{1}
\end{array}\right], \mathcal{A}^{2}\left[\begin{array}{l}
\boldsymbol{E}_{0} \\
\boldsymbol{H}_{1}
\end{array}\right], \ldots\right\}
$$

2.2 Cauer Ladder Network Representation Here, the Cauer ladder network representation is derived by using the procedure discussed in the preceding section. Before proceeding to the details, two properties of the equivalent circuit should be confirmed.

First, the separation of variables, time and space, is required in order to construct the equivalent circuit. In case of the static field, it was already carried out as $\overline{\boldsymbol{E}}_{0}=\boldsymbol{E}_{0} / v$ and $\overline{\boldsymbol{H}}_{1}=\boldsymbol{H}_{1} / i_{0}$. Thus, the electric field and magnetic field are assumed to be represented by the linear combinations (27).

$$
\boldsymbol{E}=\sum_{n=0}^{\infty} e_{2 n} \overline{\boldsymbol{E}}_{2 n}, \quad \boldsymbol{H}=\sum_{n=0}^{\infty} h_{2 n+1} \overline{\boldsymbol{H}}_{2 n+1}
$$

Here, $e_{2 n}$ and $h_{2 n+1}$ are scalar valued time function having the units $\mathrm{V}$ and $\mathrm{A}$ respectively, and the functions $\overline{\boldsymbol{E}}_{2 n}$ and $\overline{\boldsymbol{H}}_{2 n+1}$ are vector valued functions whose units are both $1 / \mathrm{m}$. Furthermore, the constants $\lambda_{2 n}$ and $\lambda_{2 n+1}$, the norms of $\overline{\boldsymbol{E}}_{2 n}$ and $\overline{\boldsymbol{H}}_{2 n+1}$ with weight functions $\sigma$ and $\mu$ respectively, are defined by the followings. The constants $R_{2 n}=1 / \lambda_{2 n}$ and $L_{2 n+1}=\lambda_{n 2+1}$ are used in the ladder network. It is assumed that the voltage $e_{2 n}$ is applied to the resistor $R_{2 n}$ and the current $h_{2 n+1}$ flows through the inductor $L_{2 n+1}$.

$$
\begin{aligned}
& \lambda_{2 n}=1 / R_{2 n}=\left\langle\sigma \overline{\boldsymbol{E}}_{2 n}, \overline{\boldsymbol{E}}_{2 n}\right\rangle \ldots \ldots \\
& \lambda_{n 2+1}=L_{2 n+1}=\left\langle\mu \overline{\boldsymbol{H}}_{2 n+1}, \overline{\boldsymbol{H}}_{2 n+1}\right\rangle .
\end{aligned}
$$

One more important requirement for the equivalent circuit is that the following Parseval's equalities must hold in the network.

$$
\begin{aligned}
& \langle\sigma \boldsymbol{E} \cdot \boldsymbol{E}\rangle=\sum_{n=0}^{\infty} e_{2 n}^{2} / R_{2 n} . \\
& \langle\mu \boldsymbol{H}, \boldsymbol{H}\rangle=\sum_{n=0}^{\infty} L_{2 n+1} i_{2 n+1}^{2}
\end{aligned}
$$

This implies that the series $\left\{\overline{\boldsymbol{E}}_{2 n}\right\}$ and $\left\{\overline{\boldsymbol{H}}_{2 n+1}\right\}$ shall be orthogonal respectively. If the orthogonality is not satisfied, the mutual inductances between inductors or contacts between resistors may arise.

The static functions $\overline{\boldsymbol{E}}_{0}, \overline{\boldsymbol{H}}_{1}$ and circuit constants $R_{0}=$ $1 / \lambda_{0}, L_{1}=\lambda_{1}$ are already obtained. Thus, by setting $n=1$, we can solve the following equation from (23).

$$
\widetilde{e}_{2 n}\left(\nabla \times \widetilde{\boldsymbol{E}}_{2 n}\right)=-\mathrm{j} \omega \mu\left(h_{2 n-1} \overline{\boldsymbol{H}}_{2 n-1}\right)
$$

Here, the upper mark $(\sim)$ is used instead of $(-)$, because $\widetilde{\boldsymbol{E}}_{2 n}$ may not be orthogonal to $\overline{\boldsymbol{E}}_{2 m}(m<n)$. Because this equation can be associated with the circuit equation $\widetilde{e}_{2 n}=$ $\mathrm{j} \omega L_{2 n-1} h_{2 n-1}$, (32) can be modified as (33).

$$
\widetilde{e}_{2 n}\left(\nabla \times \widetilde{\boldsymbol{E}}_{2 n}\right)=\mathrm{j} \omega L_{2 n-1} h_{2 n-1} \cdot\left(-\mu \overline{\boldsymbol{H}}_{2 n-1} / L_{2 n-1}\right)
$$

Then the equation after the separation of variables is derived as

$$
\nabla \times \widetilde{\boldsymbol{E}}_{2 n}=-\mu \overline{\boldsymbol{H}}_{2 n-1} / L_{2 n-1} \cdots
$$

Applying the Gram-Schmidt orthogonalization to $\widetilde{\boldsymbol{E}}_{2 n}$, the function $\overline{\boldsymbol{E}}_{2 n}$ together with the resistance $1 / R_{2 n}=$ $\left\langle\sigma \overline{\boldsymbol{E}}_{2 n}, \overline{\boldsymbol{E}}_{2 n}\right\rangle$, is obtained as the following.

$$
\overline{\boldsymbol{E}}_{2 n}=\widetilde{\boldsymbol{E}}_{2 n}-\sum_{i=0}^{n-1}\left\langle\sigma \widetilde{\boldsymbol{E}}_{2 n}, \overline{\boldsymbol{E}}_{2 i}\right\rangle \overline{\boldsymbol{E}}_{2 i} / \lambda_{2 i} \ldots \ldots \ldots \ldots
$$

Similarly to the electric field, (24) yield the following.

$$
\widetilde{h}_{2 n+1}\left(\nabla \times \widetilde{\boldsymbol{H}}_{2 n+1}\right)=\sigma e_{2 n} \overline{\boldsymbol{E}}_{2 n}
$$

Assuming the circuit equation $\widetilde{h}_{2 n+1}=e_{2 n} / R_{2 n}$, (36) is modified by the following.

$$
\widetilde{h}_{2 n+1}\left(\nabla \times \widetilde{\boldsymbol{H}}_{2 n+1}\right)=\left(e_{2 n} / R_{2 n}\right)\left(\sigma R_{2 n} \overline{\boldsymbol{E}}_{2 n}\right) \cdots \cdots
$$


Then the equation after the separation of variables is given by,

$$
\nabla \times \widetilde{\boldsymbol{H}}_{2 n+1}=\sigma \boldsymbol{R}_{2 n} \overline{\boldsymbol{E}}_{2 n}
$$

The orthogonalized functions $\overline{\boldsymbol{H}}_{2 n+1}$, together with the inductances $L_{2 n+1}=\left\langle\mu \overline{\boldsymbol{H}}_{2 n+1}, \overline{\boldsymbol{H}}_{2 n+1}\right\rangle$, are given by the following.

$$
\overline{\boldsymbol{H}}_{2 n+1}=\widetilde{\boldsymbol{H}}_{2 n+1}-\sum_{i=0}^{n-1}\left\langle\mu \widetilde{\boldsymbol{H}}_{2 n+1}, \overline{\boldsymbol{H}}_{2 i+1}\right\rangle \overline{\boldsymbol{H}}_{2 i+1} / \lambda_{2 i+1}
$$

Repeating these procedures for $n=2,3, \ldots$, the orthogonal series $\left\{\overline{\boldsymbol{E}}_{2 n}\right\}$ and $\left\{\overline{\boldsymbol{H}}_{2 n+1}\right\}$ are derived.

The inner products in the right sides of (35) and (39) are simplified as follows. Let $n$ and $i \leq n$ are fixed, then (40) is yielded by using (39), orthogonality of $\overline{\boldsymbol{H}}_{2 i+1}(i<n)$, and integration by part.

$$
\begin{aligned}
& \left\langle\sigma \widetilde{\boldsymbol{E}}_{2 n}, \overline{\boldsymbol{E}}_{2 i}\right\rangle \\
& \quad=\left\langle\sigma \widetilde{\boldsymbol{E}}_{2 n},\left(\lambda_{2 i} / \sigma\right) \nabla \times \widetilde{\boldsymbol{H}}_{2 i+1}\right\rangle \\
& \quad=\lambda_{2 i}\left\langle\nabla \times \widetilde{\boldsymbol{E}}_{2 n}, \widetilde{\boldsymbol{H}}_{2 i+1}\right\rangle \\
& \quad=-\frac{\lambda_{2 i}}{\lambda_{2 n-1}}\left\langle\mu \overline{\boldsymbol{H}}_{2 n-1}, \widetilde{\boldsymbol{H}}_{2 i+1}\right\rangle=\left\{\begin{array}{cc}
0 & (i<n-1) \\
-\lambda_{2 n-2} & (i=n-1)
\end{array}\right.
\end{aligned}
$$

Similarly, by using (35), orthogonality of $\overline{\boldsymbol{E}}_{2 i}(i<n)$ and integration by part, the following is obtained.

$$
\begin{aligned}
\left\langle\mu \widetilde{\boldsymbol{H}}_{2 n+1}, \overline{\boldsymbol{H}}_{2 i+1}\right\rangle \\
=\left\langle\mu \widetilde{\boldsymbol{H}}_{2 n+1},-\left(\lambda_{2 i+1} / \mu\right) \nabla \times \widetilde{\boldsymbol{E}}_{2 i+2}\right\rangle \\
=-\lambda_{2 i+1}\left\langle\nabla \times \widetilde{\boldsymbol{H}}_{2 n+1}, \widetilde{\boldsymbol{E}}_{2 i+2}\right\rangle \\
=-\frac{\lambda_{2 i+1}}{\lambda_{2 n}}\left\langle\sigma \overline{\boldsymbol{E}}_{2 n}, \widetilde{\boldsymbol{E}}_{2 i+2}\right\rangle=\left\{\begin{array}{cc}
0 & (i<n-1) \\
-\lambda_{2 n-1} & (i=n-1)
\end{array}\right.
\end{aligned}
$$

Remember that $\nabla \cdot(\boldsymbol{E} \times \boldsymbol{H})=(\nabla \times \boldsymbol{E}) \cdot \boldsymbol{H}-\boldsymbol{E} \cdot(\nabla \times \boldsymbol{H})$ and the divergence theorem. In the integration by part in (40) and (41), the surplus of the definite integral at the boundary $\partial \Omega$, are assumed to be zero as (42) and (43).

$$
\begin{gathered}
\int_{\partial \Omega}\left(\widetilde{\boldsymbol{E}}_{2 n} \times \widetilde{\boldsymbol{H}}_{2 i+1}\right) \cdot \boldsymbol{n} \mathrm{d} S=0 \quad(i<n) \ldots \ldots \ldots \ldots \\
\int_{\partial \Omega}\left(\widetilde{\boldsymbol{H}}_{2 n+1} \times \widetilde{\boldsymbol{E}}_{2 i+2}\right) \cdot \boldsymbol{n} \mathrm{d} S=0 \quad(i<n) \ldots \ldots \ldots
\end{gathered}
$$

The integrands in (42) and (43) are the energy flux called Poynting vectors. Thus, this assumption is quite natural because the external energy exchange is restricted only on the electrodes $\Gamma_{\mathrm{B} 0}$ and $\Gamma_{\mathrm{B} 1}$.

After all, (35) and (39) are simplified as the following. The procedure for obtaining the series $\left\{\overline{\boldsymbol{E}}_{2 n}\right\}$ and $\left\{\overline{\boldsymbol{H}}_{2 n+1}\right\}$ are summarized in Fig. 5.

$$
\begin{aligned}
& \overline{\boldsymbol{E}}_{2 n}=\widetilde{\boldsymbol{E}}_{2 n}+\overline{\boldsymbol{E}}_{2 n-2} \cdots \\
& \overline{\boldsymbol{H}}_{2 n+1}=\widetilde{\boldsymbol{H}}_{2 n+1}+\overline{\boldsymbol{H}}_{2 n-1}
\end{aligned}
$$

Hereafter, the equivalent circuit, having the variables $e_{2 n}$ and $h_{2 n+1}$, is derived. If the Maxwell's equations is valid for

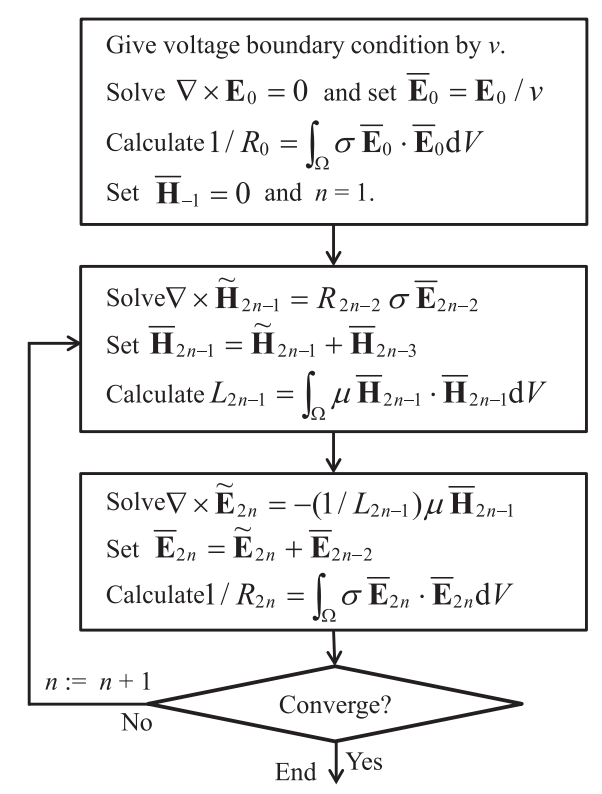

Fig. 5. Generation of Cauer ladder network

(27), it is necessary that following equations hold for arbitrary $\overline{\boldsymbol{E}}_{2 m}$ and $\overline{\boldsymbol{H}}_{2 m+1}$.

$$
\begin{aligned}
\langle\nabla & \left.\times \boldsymbol{H}-\sigma \boldsymbol{E}, \overline{\boldsymbol{E}}_{2 m}\right\rangle \\
& =\left\langle\nabla \times \sum_{n=0}^{\infty} h_{2 n+1} \overline{\boldsymbol{H}}_{2 n+1}-\sigma \sum_{n=0}^{\infty} e_{2 n} \overline{\boldsymbol{E}}_{2 n}, \overline{\boldsymbol{E}}_{2 m}\right\rangle \\
& =\sum_{n=0}^{\infty} h_{2 n+1}\left\langle\nabla \times \overline{\boldsymbol{H}}_{2 n+1}, \overline{\boldsymbol{E}}_{2 m}\right\rangle-\lambda_{2 m} e_{2 m}=0 \\
\langle\nabla & \left.\times \boldsymbol{E}+\frac{\mathrm{d}}{\mathrm{d} t} \mu \boldsymbol{H}, \overline{\boldsymbol{H}}_{2 m+1}\right\rangle \ldots \ldots \ldots \\
& =\left\langle\nabla \times \sum_{n=0}^{\infty} e_{2 n} \overline{\boldsymbol{E}}_{2 n}+\frac{\mathrm{d}}{\mathrm{d} t} \mu \sum_{n=0}^{\infty} h_{2 n+1} \overline{\boldsymbol{H}}_{2 n+1}, \overline{\boldsymbol{H}}_{2 m+1}\right\rangle \\
& =\sum_{n=0}^{\infty} e_{2 n}\left\langle\nabla \times \overline{\boldsymbol{E}}_{2 n}, \overline{\boldsymbol{H}}_{2 m+1}\right\rangle+\frac{\mathrm{d}}{\mathrm{d} t} \lambda_{2 m+1} h_{2 m+1}=0
\end{aligned}
$$

On the other hand, (34), (38), (44), and (45) yield the followings.

$$
\begin{aligned}
\langle\nabla & \left.\times \overline{\boldsymbol{H}}_{2 n+1}, \overline{\boldsymbol{E}}_{2 m}\right\rangle \\
& =\left\langle\nabla \times\left(\widetilde{\boldsymbol{H}}_{2 n+1}+\overline{\boldsymbol{H}}_{2 n-1}\right), \overline{\boldsymbol{E}}_{2 m}\right\rangle \\
& =\left\langle\frac{\sigma}{\lambda_{2 n}} \overline{\boldsymbol{E}}_{2 n}+\nabla \times \overline{\boldsymbol{H}}_{2 n-1}, \overline{\boldsymbol{E}}_{2 m}\right\rangle=\ldots \\
& =\left\langle\sum_{i=0}^{n} \frac{\sigma}{\lambda_{2 i}} \overline{\boldsymbol{E}}_{2 i}, \overline{\boldsymbol{E}}_{2 m}\right\rangle=\left\{\begin{array}{ll}
0 & (n<m) \\
1 & (n \geq m)
\end{array} \ldots \ldots\right. \\
\langle\nabla & \left.\times \overline{\boldsymbol{E}}_{2 n}, \overline{\boldsymbol{H}}_{2 m+1}\right\rangle \\
& =\left\langle\nabla \times\left(\widetilde{\boldsymbol{E}}_{2 n}+\overline{\boldsymbol{E}}_{2 n-2}\right), \overline{\boldsymbol{H}}_{2 m+1}\right\rangle \\
& =\left\langle-\frac{\mu}{\lambda_{2 n-1}} \overline{\boldsymbol{H}}_{2 n-1}+\nabla \times \overline{\boldsymbol{E}}_{2 n-2}, \overline{\boldsymbol{H}}_{2 m+1}\right\rangle=\ldots \\
& =\left\langle-\sum_{i=1}^{n} \frac{\mu}{\lambda_{2 i-1}} \overline{\boldsymbol{H}}_{2 i-1}, \overline{\boldsymbol{H}}_{2 m+1}\right\rangle=\left\{\begin{array}{cc}
0 & (n \leq m) \\
-1 & (n>m)
\end{array}\right.
\end{aligned}
$$


From (46), (47), (48), and (49), the following equations are derived. The static network shown in Fig. 2 is extended to the Cauer ladder network shown in Fig. 3.

$$
\begin{aligned}
& e_{2 m}=R_{2 m} \sum_{n=m}^{\infty} h_{2 n+1} . \cdots \\
& \frac{\mathrm{d}}{\mathrm{d} t} h_{2 m+1} L_{2 m+1}=\sum_{n=m+1}^{\infty} e_{2}
\end{aligned}
$$

Once the constants of the CLN and distribution functions are obtained, there is no need to perform the finite element calculations in transient analysis. The magnetic field at arbitrary point of time is expressed by (27). The coefficients $e_{2 n}$ and $h_{2 n+1}$ are easily obtained by circuit equations, usually solved by existent circuit simulators.

2.3 State Equations of Truncated Networks The finite length truncation of the ladder network is required for the practical applications. There are two types of the truncation, resistive and inductive termination as shown in Fig. 6. When the frequency becomes infinitely large, the impedance of the network with resistive termination is resistive, while that of inductive termination is inductive.

The state equation for the CLN with inductive termination is derived as follows. Let the CLN is truncated to $N$ stages, which implies that the resistor $R_{2 N}$ is removed from the network. The circuit laws yield the followings.

$$
\begin{aligned}
& L_{2 n+1} \frac{\mathrm{d}}{\mathrm{d} t} h_{2 n+1}=v-\sum_{m=0}^{n} R_{2 m} h_{2 m} \quad(n=0, \ldots, N-1) \\
& h_{2 m}=\sum_{i=m}^{N-1} h_{2 i+1} \quad(m=0, \ldots, N-1)
\end{aligned}
$$

Then the state equation for the CLN is provided by the followings.

$$
\begin{aligned}
& \boldsymbol{L}_{N} \frac{\mathrm{d}}{\mathrm{d} t} \boldsymbol{h}+\boldsymbol{R}_{N} \boldsymbol{h}=\mathbf{1}_{N} v \cdots \cdots \cdots \cdots \\
& \boldsymbol{h}_{N}=\left[\begin{array}{c}
h_{1} \\
h_{3} \\
\vdots \\
h_{2 N-1}
\end{array}\right], \mathbf{1}_{N}=\left[\begin{array}{c}
1 \\
1 \\
\vdots \\
1
\end{array}\right], \boldsymbol{U}_{N}=\left[\begin{array}{cccc}
1 & 1 & \cdots & 1 \\
0 & 1 & \ddots & \vdots \\
\vdots & \vdots & \ddots & 1 \\
0 & 0 & \cdots & 1
\end{array}\right], \ldots(54), \ldots \cdots \cdots(53) \\
& \boldsymbol{L}_{N}=\operatorname{diag}\left[\begin{array}{llll}
L_{1} & L_{3} & \cdots & L_{2 N-1}
\end{array}\right], \\
& \boldsymbol{R}_{N}=\boldsymbol{U}_{N}^{\mathrm{T}} \operatorname{diag}\left[\begin{array}{llll}
R_{0} & R_{2} & \cdots & R_{2 N-2}
\end{array}\right] \boldsymbol{U}_{N} .
\end{aligned}
$$

From (53), the expression by transfer admittance $\boldsymbol{Y}_{I}$ for the inductive termination is given by the following.

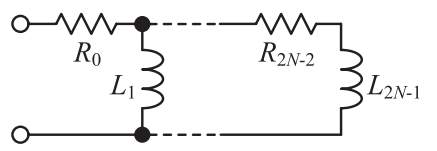

(a) Inductive termination

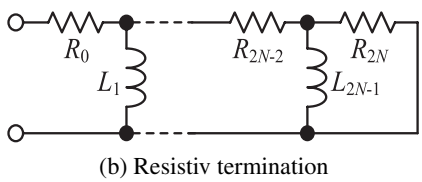

Fig. 6. Termination of ladder network

$$
\boldsymbol{h}=\boldsymbol{Y}_{I} v, \quad \boldsymbol{Y}_{I}=\left(\boldsymbol{R}_{N}+s \boldsymbol{L}_{N}\right)^{-1} 1_{N}
$$

Replacing $\boldsymbol{L}_{N}$ and $\boldsymbol{R}_{N}$ with

$$
\begin{aligned}
\overline{\boldsymbol{L}}_{N+1} & =\operatorname{diag}\left[\begin{array}{lllll}
L_{1} & L_{3} & \cdots & L_{2 N-1} & 0
\end{array}\right], \\
\boldsymbol{R}_{N+1} & =\boldsymbol{U}_{N+1}^{\mathrm{T}} \operatorname{diag}\left[\begin{array}{lllll}
R_{0} & R_{2} & \cdots & R_{2 N-2} & R_{2 N}
\end{array}\right] \boldsymbol{U}_{N+1},
\end{aligned}
$$

The transfer admittance $\boldsymbol{Y}_{R}$ for the resistive termination is given by the following,

$$
\begin{aligned}
& \boldsymbol{h}=\boldsymbol{Y}_{R} v, \\
& \boldsymbol{Y}_{R}=\left[\boldsymbol{M}_{N_{\mathbf{l}}^{\prime}}^{\prime} \mathbf{1}_{N}\right]\left(\boldsymbol{R}_{N+1}+s \overline{\boldsymbol{L}}_{N+1}\right)^{-1} \mathbf{1}_{N+1} \cdots
\end{aligned}
$$

where $\boldsymbol{M}_{N}$ is the $N$-dimensional unit matrix.

The effects of these two terminations to the magnetic fields and the magnetic forces will be estimated in section 4 .

\section{Magnetic Force}

3.1 Magnetic Force by Maxwell's Tensor Maxwell stress tensor in eddy-current field is defined by (58). It is assumed that the coordinate of this tensor lies in in the air.

$$
\boldsymbol{T}=\frac{\mu_{0}}{2}\left[\begin{array}{ccc}
H_{x}^{2}-H_{y}^{2}-H_{z}^{2} & 2 H_{x} H_{y} & 2 H_{x} H_{z} \\
2 H_{y} H_{x} & H_{y}^{2}-H_{x}^{2}-H_{z}^{2} & 2 H_{y} H_{z} \\
2 H_{z} H_{x} & 2 H_{z} H_{y} & H_{z}^{2}-H_{x}^{2}-H_{y}^{2}
\end{array}\right]
$$

Using (27), we obtain the following representation, the quadratic form of the current $h_{2 n+1}$ of the network.

$$
\begin{aligned}
& \boldsymbol{T}=\frac{\mu_{0}}{2} \sum_{n=0}^{\infty} \sum_{m=0}^{\infty} h_{2 n+1} h_{2 m+1} \overline{\boldsymbol{T}}_{n, m} \\
& \overline{\boldsymbol{T}}_{n, m}=\overline{\boldsymbol{T}}_{m, n}^{\mathrm{T}} \\
& =\left[\begin{array}{lll}
\bar{H}_{2 n+1, x} \bar{H}_{2 m+1, x} & \overline{\bar{H}}_{2 n+1, x} \bar{H}_{2 m+1, y} & 2 \bar{H}_{2 n+1, x} \bar{H}_{2 m+1, z} \\
-\bar{H}_{2 n+1, y} \bar{H}_{2 m+1, y} & \bar{H}_{2 n+1, z} \bar{H}_{2 m+1, z} & \bar{H}_{2 n+1, y} \bar{H}_{2 m+1, y} \\
2 \bar{H}_{2 n+1, y} \bar{H}_{2 m+1, x} & -\bar{H}_{2 n+1, x} \bar{H}_{2 m+1, x} & 2 \bar{H}_{2 n+1, y} \bar{H}_{2 m+1, z} \\
& -\bar{H}_{2 n+1, z} \bar{H}_{2 m+1, z} & \bar{H}_{2 n+1, z} \bar{H}_{2 m+1, z} \\
2 \bar{H}_{2 n+1, z} \bar{H}_{2 m+1, x} & 2 \bar{H}_{2 n+1, z} \bar{H}_{2 m+1, y} & -\bar{H}_{2 n+1, x} \bar{H}_{2 m+1, x} \\
& & -\bar{H}_{2 n+1, y} \bar{H}_{2 m+1, y}
\end{array}\right]
\end{aligned}
$$

As illustrated in Fig. 2, we assume that the iron piece pulled by the electromagnet is covered by a closed surface $\Gamma_{S}$. Then the applied force to the iron piece is given by the followings. The block diagram from input coil voltage $v$ to the magnetic force $\boldsymbol{F}$ is given by Fig. 7.

$$
\begin{gathered}
\boldsymbol{F}=\int_{\Gamma_{S}} \boldsymbol{T} \boldsymbol{n} \mathrm{d} S=\frac{\mu_{0}}{2} \sum_{n=0}^{\infty} \sum_{m=0}^{\infty} h_{2 n+1} h_{2 m+1} \overline{\boldsymbol{F}}_{n, m} \ldots \\
\overline{\boldsymbol{F}}_{m, n}=\int_{\Gamma_{S}} \overline{\boldsymbol{T}}_{m, n} \boldsymbol{n} \mathrm{d} S \\
v \rightarrow \boldsymbol{Y}_{L(R)} \underset{\frac{\boldsymbol{\mu _ { 0 }}}{2}\left[\overline{\boldsymbol{F}}_{n, m}\right]}{\longrightarrow} \rightarrow \boldsymbol{F}
\end{gathered}
$$

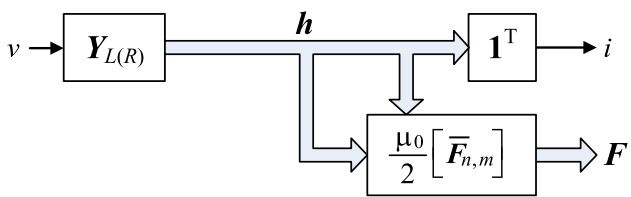

Fig. 7. Block diagram of magnetic force 


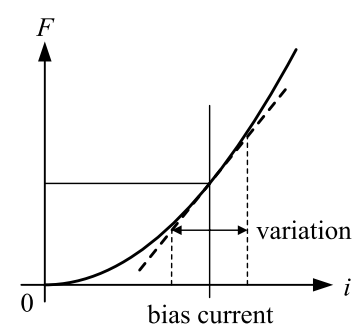

Fig. 8. Linearization of the magnetic force

It is should be noted that instead of the Maxwell stress tensor, the nodal force method ${ }^{(14)}$ can be used to obtain the force coefficients (60) when the magnetic field is solved by FE analysis. However, this article adopt the Maxwell tensor just because its popularity.

3.2 Linearized Transfer Function for the Magnetic Force Especially for the use of magnetic levitations, the electromagnet often operated around the preset bias magnetic field as shown in Fig. 8. Thus the linear approximation of the magnetic force is required. The transfer function for linearized magnetic force around the bias field is obtained as followings.

Let the voltage $v=v_{B}$ be the bias voltage of the excitation windings. The DC bias current flows only through the first inductor of the CLN. This yields the followings.

$$
\begin{aligned}
& i_{B}=v_{B} / R_{0}, \\
& \boldsymbol{h}_{B}=\left[\begin{array}{llll}
i_{B} & 0 & 0 & \ldots
\end{array}\right]
\end{aligned}
$$

From (60) and (61), we obtain bias force as following.

$$
\boldsymbol{F}_{B}=\frac{\mu_{0}}{2} i_{B}^{2} \overline{\boldsymbol{F}}_{0,0}
$$

If the exciting voltage varies, the current in the CLN varies as,

$$
\begin{aligned}
& v=v_{B}+\Delta v \\
& \boldsymbol{h}=\boldsymbol{h}_{B}+\Delta \boldsymbol{h}=\left[\begin{array}{lll}
i_{B} & 0 & \ldots
\end{array}\right]^{\mathrm{T}}+\left[\begin{array}{lll}
\Delta h_{1} & \Delta h_{3} & \ldots
\end{array}\right]^{\mathrm{T}},
\end{aligned}
$$

Here, the current variation $\Delta \boldsymbol{h}$ is given by the state equation (53) with the input voltage $\Delta v$. From (60) and (63), then the magnetic force (63) is derived.

$$
\begin{aligned}
\boldsymbol{F}= & \frac{\mu_{0}}{2} i_{B}^{2} \overline{\boldsymbol{F}}_{0,0}+\frac{\mu_{0}}{2} i_{B} \sum_{n=0}^{\infty} \Delta h_{2 n+1}\left(\overline{\boldsymbol{F}}_{n, 0}+\overline{\boldsymbol{F}}_{0, n}\right) \\
& +\frac{\mu_{0}}{2} \sum_{n=0}^{\infty} \sum_{m=0}^{\infty} \Delta h_{2 n+1} \Delta h_{2 m+1} \overline{\boldsymbol{F}}_{n, m} \ldots \ldots \ldots \ldots
\end{aligned}
$$

Neglecting the second order terms of $\Delta \boldsymbol{h}$ and using (62), the variation of the magnetic force is obtained as

$$
\begin{aligned}
& \boldsymbol{F} \approx \boldsymbol{F}_{B}+\Delta \boldsymbol{F} \\
& \Delta \boldsymbol{F}=\frac{\mu_{0}}{2} i_{B} \sum_{n=0}^{\infty} \Delta h_{2 n+1}\left(\overline{\boldsymbol{F}}_{n, 0}+\overline{\boldsymbol{F}}_{0, n}\right) .
\end{aligned}
$$

Figure 9 shows the block diagram of the linearized magnetic force. Note that the amplification of the force is proportional to the bias current $i_{B}=v_{B} / R_{1}$.

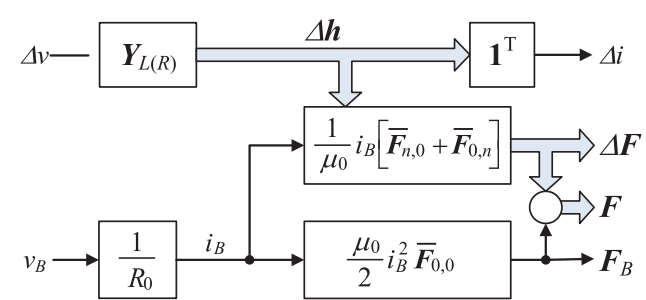

Fig. 9. Block diagram of linearized magnetic force

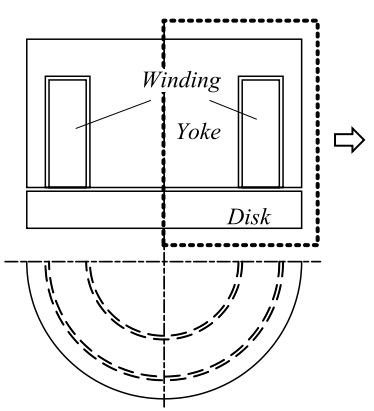

(a) Drawing (b) FE mesh division

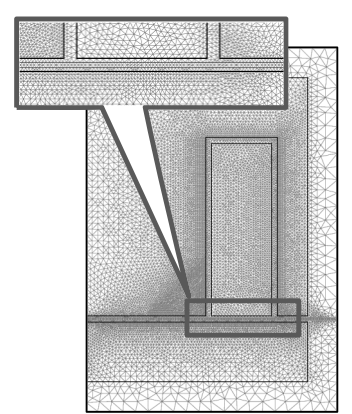

Fig. 10. Electromagnet

\section{Numerical Results}

\subsection{Electromagnet and its CLN Representation}

The electromagnet shown in Fig. 10(a) is analyzed. The electromagnet has a cylindrical shape with $40 \mathrm{~mm}$ height and $74 \mathrm{~mm}$ diameter. A multi-turn winding is included in the electromagnet. A disk with $10 \mathrm{~mm}$ thickness is set toward to the electromagnet with $1 \mathrm{~mm}$ air gap. The conductivity of the magnetic material is $8.0 \mathrm{E} 6 \mathrm{~S} / \mathrm{m}$, and the relative permeability is 500. The mesh division for FE analysis is illustrated in Fig. 10(b). The minimum size of an element is $0.25 \mathrm{~mm}$, which corresponds to the skin depth at $1 \mathrm{kHz}$. The surface of the material is divided finely. All the simulations are performed by Freefem $++{ }^{(15)}$.

The FE analysis is carried out with $\boldsymbol{A}-\boldsymbol{\varphi}$ formulation, which is based on the vector potential $\boldsymbol{A}$ and scalar potential $\boldsymbol{\varphi}$. The FE formulation is based on 3-dimensional cylindrical coordinate. The vector potential $\boldsymbol{A}$ is the unknown variable for $\mathrm{FE}$ analysis. The Dirichlet boundary condition $\boldsymbol{A}=0$ and $\varphi=0$ is supposed on $\partial \Omega$. This condition makes the assumptions (42) and (43) valid, because the identity $\boldsymbol{E}=-\partial \boldsymbol{A} / \partial t-\nabla \varphi=$ 0 holds on $\partial \Omega$.

Firstly the distribution functions $\overline{\boldsymbol{E}}_{2 n}, \overline{\boldsymbol{H}}_{2 n+1}$ and the circuit constants $R_{2 n}$ and $L_{2 n+1}$ are calculated. Several distribution functions $\overline{\boldsymbol{E}}_{2 n}$ at the beginning are shown in Fig. 11(a)-(e) by the intensity. White color means positive and black means negative direction perpendicular to the cross section. Figure 11(f)-(i) shows the lines of magnetic flux, which are equipotentials of $r \overline{\boldsymbol{A}}_{2 n+1}$. The vector potential $\overline{\boldsymbol{A}}_{2 n+1}$ is defined by $\overline{\boldsymbol{H}}_{2 n+1}=v \nabla \times \overline{\boldsymbol{A}}_{2 n+1}$. The difference between minimum and maximum magnitude of $r \overline{\boldsymbol{A}}_{2 n+1}$ is divided to twenty by contour lines. Table 1 shows the constants of the CLN.

In order to evaluate the truncation errors of the CLN, the magnetic field synthesized by linear combination (27) is compared with the results by complex FE analysis. The CLN is truncated to twenty-one stages with resistive termination. The magnetic fluxes by the CLN at $10 \mathrm{~Hz}, 100 \mathrm{~Hz}$ and $1 \mathrm{kHz}$ 


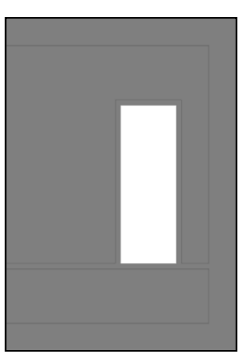

(a) $\overline{\mathbf{E}}_{0}$

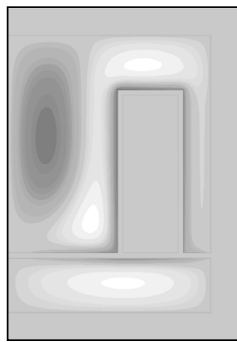

(d) $\overline{\mathbf{E}}_{6}$

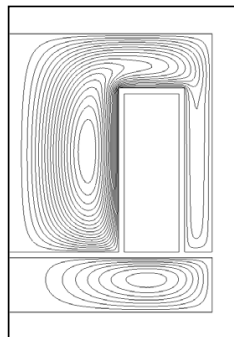

(g) $r \overline{\mathbf{A}}_{3}$

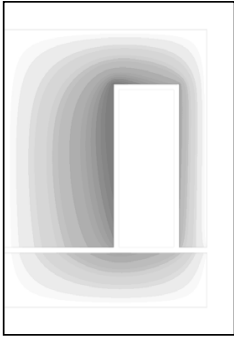

(b) $\overline{\mathbf{E}}_{2}$

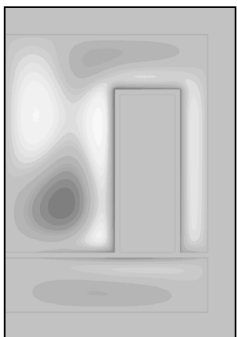

(e) $\overline{\mathbf{E}}_{8}$

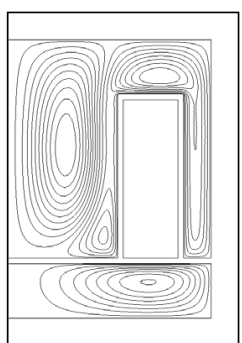

(h) $r \overline{\mathbf{A}}_{5}$

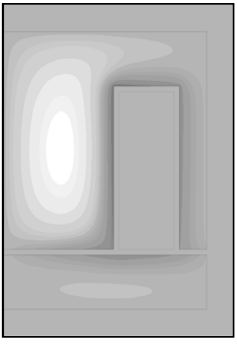

(c) $\overline{\mathbf{E}}_{4}$

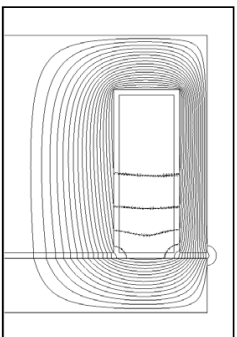

(f) $r \overline{\mathbf{A}}_{1}$

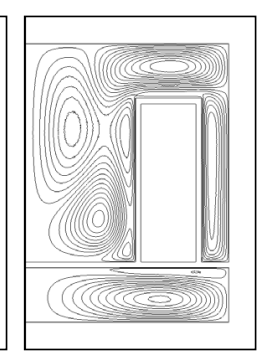

(i) $r \overline{\mathbf{A}}_{7}$
Fig. 11. Distribution functions

Table 1. Constants of CLN

\begin{tabular}{c|c|c|c}
\hline \multicolumn{2}{c|}{ Resistance $[\Omega]$} & \multicolumn{2}{c}{ Inductance $[\mathrm{H}]$} \\
\hline \hline$R_{0}$ & 1.04 & $L_{1}$ & 0.0803 \\
\hline$R_{2}$ & 5.53 & $L_{3}$ & 0.282 \\
\hline$R_{3}$ & 6.38 & $L_{5}$ & 0.207 \\
\hline$R_{4}$ & 9.30 & $L_{7}$ & 0.158 \\
\hline
\end{tabular}

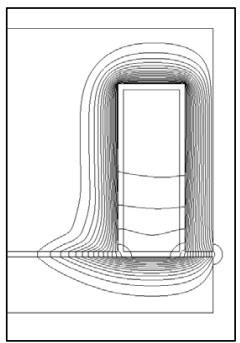

(a) $10 \mathrm{~Hz}$

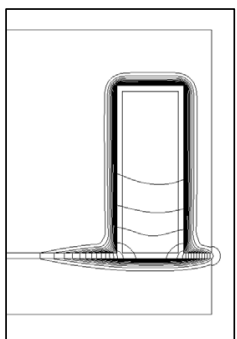

(b) $100 \mathrm{~Hz}$

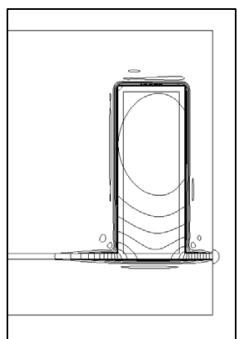

(c) $1 \mathrm{kHz}$
Fig. 12. CLN representation at 21 stages

are shown in Fig. 10. The results by the complex analysis are shown in Fig. 11. The errors at 10 and $100 \mathrm{~Hz}$ are little. Small difference is observed at $1 \mathrm{kHz}$ in the core and the disk near the surfaces, though the error seems still small.

The magnetic field by the inductive termination is also calcutated and compared with the resistive termination. Figure 14 shows comparison of the magnetic flux at $1 \mathrm{kHz}$ by the network of twenty-one stages. The difference between them can not be ignored, although the difference of the fluxes at the air gap is small. This difference can be explained as followings.

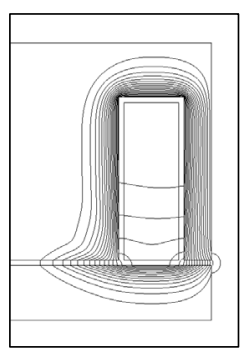

(a) $10 \mathrm{~Hz}$

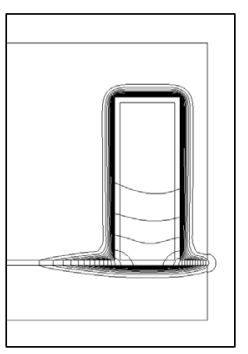

(b) $100 \mathrm{~Hz}$

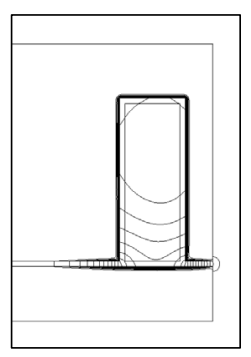

(c) $1 \mathrm{kHz}$
Fig. 13. Complex FE analyses

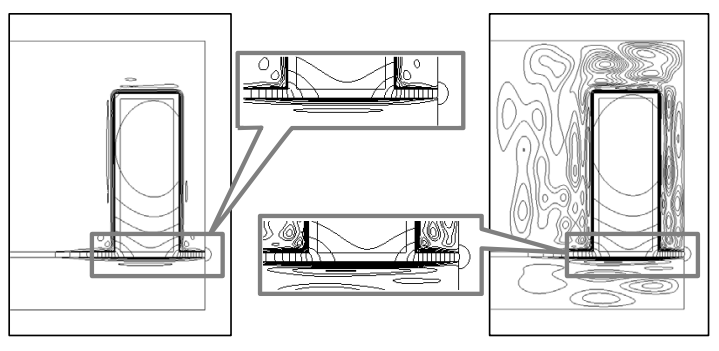

(a) resistive termination

(b) Inductive tremination

Fig. 14. Comparison of terminations at $1 \mathrm{kHz}$

Table 2. Coefficients for the force

\begin{tabular}{c|r}
\hline$\left(\overline{\mathbf{F}}_{0,0}\right)_{z}$ & $4.197 \mathrm{E}+07$ \\
\hline$\left(\overline{\mathbf{F}}_{0,0}+\overline{\mathbf{F}}_{0,0}\right)_{z}$ & $8.394 \mathrm{E}+07$ \\
\hline$\left(\overline{\mathbf{F}}_{1,0}+\overline{\mathbf{F}}_{0,1}\right)_{z}$ & $-1.771 \mathrm{E}+06$ \\
\hline$\left(\overline{\mathbf{F}}_{2,0}+\overline{\mathbf{F}}_{0,2}\right)_{z}$ & $-3.263 \mathrm{E}+06$ \\
\hline$\left(\overline{\mathbf{F}}_{3,0}+\overline{\mathbf{F}}_{0,3}\right)_{z}$ & $-1.060 \mathrm{E}+06$ \\
\hline$\left(\overline{\mathbf{F}}_{4,0}+\overline{\mathbf{F}}_{0,4}\right)_{z}$ & $-4.263 \mathrm{E}+06$ \\
\hline
\end{tabular}

At high frequency range, the current distribution reaches to the latter part of the network, while the current flows only first several elements of the network at low frequency. Thus the effect by the truncation is significant at the high frequency region. When the inductive termination is chosen, the current forward to the truncated networks lose a place to go. Then, this surplus current flows into last several inductors. If the resistive termination is adopted, this surplus current partly flows to the terminal resistor. And the effect of the truncation appeared in the magnetic field is reduced.

4.2 Magnetic Force Using the distribution functions derived above, the electromagnetic force is calculated. Table 2 is shown first several coefficients for each current $\Delta h_{2 n+1}$ appeared in (64) and Fig. 9. It is interesting that all of the coefficients in high order are negative. Observing the flux near the air gap in Fig. 9, only the DC flux in Fig. 9(f) flows vertically against the air gap, while the other flux of high order flow parallel to the air gap. This implies that only the DC flux provides the attractive force, while the flux due to the eddy current provides the repulsive force, bringing the deterioration of the total magnetic force.

The frequency response of the magnetic force at the rated DC bias current is shown in Figs. 15 and 16. The resistive and inductive terminations are adopted respectively and the number of stages of the CLN varies from 1 to 21 . The electromagnet is driven by current source, because the electromagnet is often controlled by current controlled drivers. The complex analysis is also performed by existent commercial package. These are indicated by triangle points in Figs. 15 


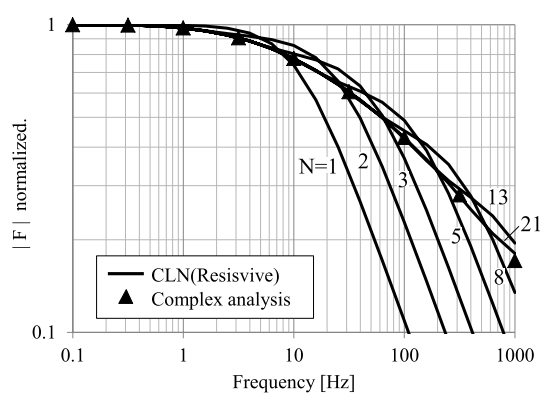

(a) Gain

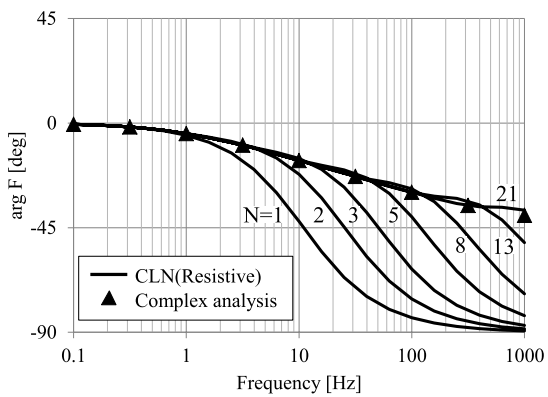

(b) Phase

Fig. 15. Frequency response of the force by resistive termination

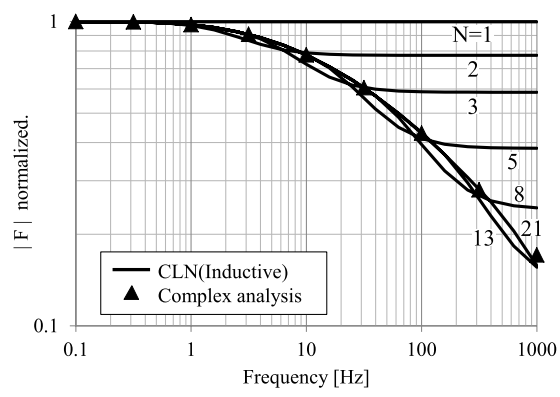

(a) Gain

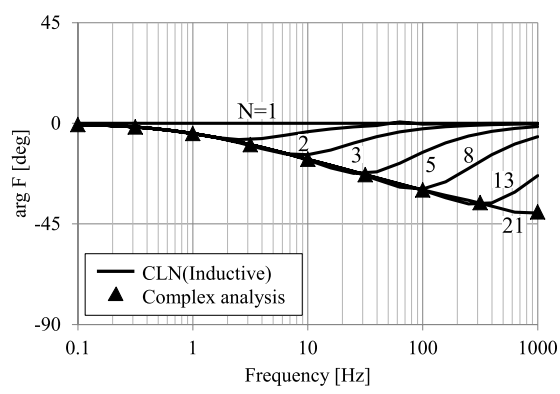

(b) Phase

Fig. 16. Frequency response of the force by inductive termination

and 16.

The result shows that twenty-one or more stages are required to guarantee the accuracy of the estimated force at $1 \mathrm{kHz}$ in both of the terminations. It is seen that the Bode plot of proposed method approaches to the results by conventional method as the number of stages of the CLN increases.

In resistive termination, the gain at high frequency is decreased about $-20 \mathrm{~dB} /$ decade, while the phase lag approaches to -90 degree. In the inductive termination, the gain at high frequency keeps the value at corner frequency, while the phase lag returns to zero degree. Thus, the terminations and the number of the stages must be carefully chosen when the

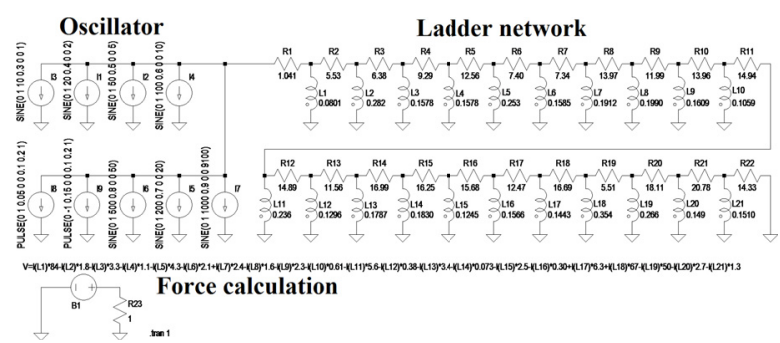

(a) Schematic for the realtime simulation

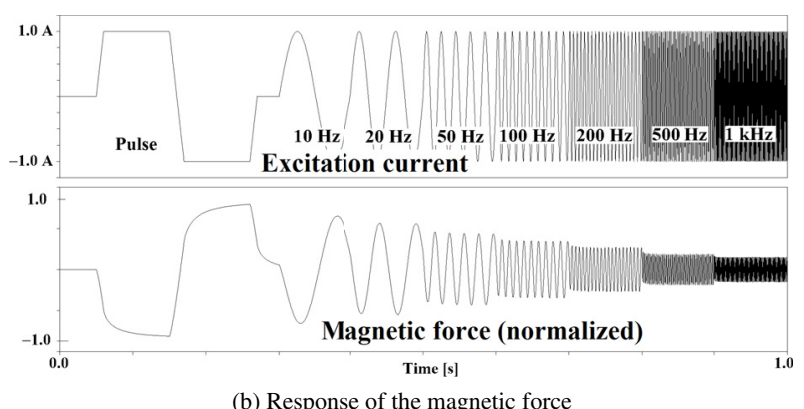

Fig. 17. Real-time simulation by Spice circuit simulator

method is applied to the control systems like in Fig. 1.

If the magnetic material is linear and its shape is simple, such as a plane or a cylinder, the number of the stages required for approximation can be already derived analytically ${ }^{(8)(9)(16)}$. There, the maximum effective frequency is proportional to the fourth power of the number of stages. However, it is difficult to obtain the number of stages in advance if the shape is not simple. Practically, the number of stage can be chosen to meet with the results obtained by the complex analyses.

The real-time simulation is performed to clarify the advantage of the proposed method. Here, the magnet is driven current source, whose waveform is two alternate pulse followed by sinusoidal wave whose frequency is swept from $10 \mathrm{~Hz}$ to $1 \mathrm{kHz}$. The circuit schematics and the results are shown in Fig. 17.

The CPU time required for generating the distribution functions and circuit constants of 21 stages CLN is about $88.1 \mathrm{~s}, 4.2 \mathrm{~s}$ per one FE analysis, while the real time simulation by the equivalent circuit is achieved by $0.94 \mathrm{~s}$ actual CPU time for the $1.0 \mathrm{sec}$ period of the simulation. Thus the total simulation is about $90 \mathrm{~s}$. The CPU time for one FE analysis is supposed to be about $4.2 \mathrm{~s}$. The discretized time for the realtime simulation must be taken at most the half of the period of the maximum frequency $1 \mathrm{kHz}$. If one perform the real-time simulation by using the FE analyses, at least $8,400 \mathrm{~s}, 4.2 \mathrm{~s}$ multiplied by 2,000 , is required for the calculation, which is 93 times larger than the proposed method.

\section{Concluding Remarks}

The linearized transfer function for the magnetic force was derived theoretically by using the CLN representations. The simulation results show that this method is adequate and practical. However, the authors recognize that there is still room for the improvement of this method on the high frequency convergence, or realization by more small number of the network element. They are unsolved subjects by now.

This paper focused on the magnetic force when the air gap 
is kept constant and the materials are linear. The implementation of the non-linearity may be the future work. Besides, one more important characteristic of the electromagnet is the displacement stiffness, the magnetic force when the air gap is varied and the current kept constant. In such case, the magnetic field is determined not only by the exciting current but also by the displacement of the air gap. The authors think that the CLN representation can also be applicable to this problem. It will be revealed in near future.

\section{References}

(1) E. Swanson, L. Hawkins, and A. Masala: "New Active Magnetic Bearing Requirements for Compressors in API 617 Eighth Edition", Proc. 43rd Turbomachinery Symposium, Sep. 22-25 Houston, Texas (2014)

( 2 ) Y. Sun, Y. Ho, and L. Yu: "Dynamic Stiffnesses of Active Magnetic Thrust Bearing Including Eddy-Current Effects", IEEE Trans. Magn., Vol.45, pp.139-149 (2009)

( 3 ) B. Han and S. Zheng: "Modeling and Analysis of Coupling Performance of Dynamic Stiffness Models for a Novel Combined Radial-Axial Hybrid Magnetic Bearing", Mathematical Problems in Engineering, Vol.2014, Article ID 340140, Hindawi (2014)

( 4 ) M. Sawada, T. Tamiya, M. Ueki, K. Nakashima, M. Kataoka, M. Kuroda, and Y. Shindo: "Frequency domain analysis of magnetic bearings and its application to the design of controller", IEEJ Trans. IA, Vol.132, pp.1131-1140 (2012) (In Japanese)

( 5 ) A. Kameari, H. Ebrahimi, K. Sugahara, Y. Shindo, and T. Matsuo: "Cauer Ladder Network Representation of Eddy-Current Fields for Model Order Reduction Using Finite Element Method", IEEE TMAG (To be published)

( 6 ) Y. Shindo, A. Kameari, and T. Matsuo: "Efficient circuit representation of eddycurrent fields", COMPEL, Int'l J. Computation and Mathematics in Electrical and Electronic Eng., Vol.36, No.5, pp.1457-1473 (2017)

( 7 ) Y. Shindo and O. Noro: "Simple Circuit Simulation Models for Eddy Current in Magnetic Sheets and Wires", IEEJ Trans. FMS, Vol.134, No.4, pp.173181 (2014)

( 8 ) Y. Shindo, T. Miyazaki, and T. Matsuo: "Cauer Circuit Representation of the Homogenized Eddy-Current Field Based on the Legendre Expansion for a Magnetic Sheet", IEEE Trans. Magn., Vol.52, \#6300504 (2016)

( 9 ) Y. Shindo, A. Kameari, and T. Matsuo: "High Frequency Nonlinear Modeling of Magnetic Sheets using Polynomial Expansions for Eddy-current Fields", IEEJ Trans. PE, Vol.137, No.3, pp.162-172 (2017)

(10) F.W. Byron, Jr. and R.W. Fuller: "Mathematics of Classical and Quantum Physics", Dover, New York, pp.469-474 (1992)

(11) K. Yoshida: "Functional Analysis Sixth Ed.", Springer, Berlin, p.69, p.197 (1980)

(12) J. Liesen and Z. Strakos: "Krylov Subspace Methods-Principles and Analysis", Oxford Univ. Press, Oxford, pp.19-21 (2013)

(13) J.P. Bastos and N. Sadowski: "Electromagnetic Modeling by Finite Element Methods", CRC Press, 2003, pp.107-121 (2003)

(14) A. Kameari: "Local force calculation in 3D FEM with edge elements", Int'l J. Applied Electromagnetics in Materials, Vo1.3, pp.231-240 (1993)

(15) F. Hecht: "FreeFem++ Third Edition, Version 3.51", http://www.freefem.org/ $\mathrm{ff}++$

(16) Y. Shindo, A. Kameari, and T. Matsuo: "Efficient circuit representation of eddycurrent fields", COMPEL - The International Journal for Computation and Mathematics in Electrical and Electronic Engineering, Vol.36, No.5, pp.1457-1473 (2017)

Yuji Shindo (Member) was born in 1956. He received his B.E. de-

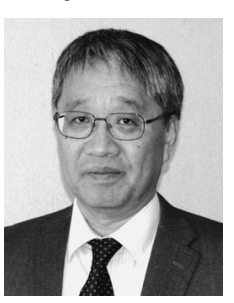
gree in electrical engineering from Okayama University in 1979 and M.E. and Dr.Eng. degrees in control engineering from Osaka University in 1981 and 1984, respectively. He joined Kawasaki Heavy Industries, Ltd. in 1984. He worked on robotics, power conversions, power systems, and high speed drives systems. $\mathrm{He}$ is a member of IEEE.

Akihisa Kameari (Member) was born in 1950. He received the B.E.

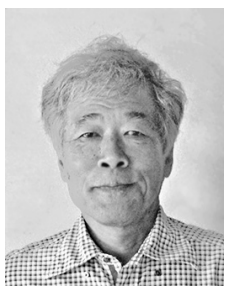
degree in physics from Kyoto University in 1973. He joined Mitsubishi Heavy Industries, Ltd. in 1973. He worked on design and analysis of nuclear fusion reactors. He joined Science Solutions International Laboratory (SSIL), Inc. as an original member of foundation. He worked on the development of software for electromagnetic field analysis and their applications. $\mathrm{He}$ is a member of IEEE.

Kengo Sugahara

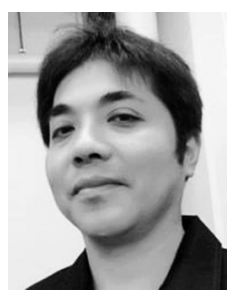

(Member) was born in 1976. He received the B.A degree in physics from Keio University in 1999, M.A. degree in physics from Kyoto University in 2001 and Dr. Eng degrees from Hosei University in 2007. He has worked for Mitsubishi Electric Corporation as a research engineer for 15 years and now a lecturer in Kindai University. His interest is a numerical method of electromagnetic field analysis and accelerator physics. He is a member of IEEE.

Tetsuji Matsuo

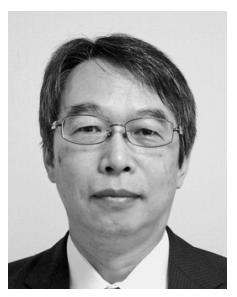

(Member) was born in 1964. He received the B.E. degree in electrical engineering from Kyoto University in 1985 and M.E. and Dr.Eng. degrees in electrical engineering from Kyoto University in 1988 and 1991, respectively. He was appointed an associate professor in the Department of Electrical Engineering of Kyoto University in 2003, and a professor in 2011. His research interests include computational electromagnetics and modeling of magnetic materials. He is a member of IEEE. 